\title{
Amr M.Alnoby ${ }^{a}$, Abdallah E. Ali ${ }^{a}$, Ayman S. Yassin ${ }^{b}$, Hanan M.Fayed $^{a}$, Hasan S.Mahmoud
}

${ }^{\mathrm{a} C h e m i c a l}$ and Clinical pathology Department, Faculty of Medicine, South Valley University, Qena, Egypt.

${ }^{b}$ Microbiology and Immunology Department, Faculty of Medicine, Al-Azhar University, Assiut branch, Assiut, Egypt

${ }^{\mathrm{c}}$ Tropical Medicine and Gastroenterology Department, Faculty of medicine, South Valley University, Qena, Egypt.

\section{Abstract}

Objectives: To evaluate noninvasive fibrosis markers, retinol binding protein 4 (RBP4), and alpha-feto protein (AFP) in foreseeing liver fibrosis in persistent hepatitis $\mathrm{C}$ patients and their correlations with Fibroscan.

Materials and Method: Fifty naive CHC patients and 20 healthy age and sex-matched subjects as controls were included. Blood samples and fibroscan obtained before and 3 months after treatment with DAAs for CBC, INR, and Liver function tests, RBP4, AFP and different fibrosis markers as APRI, CDS, FIB-4, GUCI, Lok index.

Results: All patients achieved SVR (12 weeks) with anchange of liver function and decrease of liver fibrosis as measured by Fibroscan. The Fib-4 score gave the best performance and diagnostic accuracy for the prediction of the presence of significant fibrosis, then (APRI and GUCI) followed by the CDC and LOK index; and lastly RBP4 as they were efficient to differentiate significant fibrosis (moderate and severe) liver scarring (F2-F3) from mild fibrosis (F0-F1). However; the accuracy of RBP4 improved with a higher fibrosis grade and thus can differentiate the presence or absence of severe (F3-F4) from mild \& moderate (F1-F2) liver scarring with $93.75 \%$ for PPV and NPV but with a poor prediction of the absence of significant fibrosis (F1 versus F2-F3), while LOK score was excellent in the prediction of the absence of significant fibrosis (F1 versus F2-F3).

Conclusions: FIB-4, APRI, GUCI, CDS, and Lok index were accurate to predict the fibrosis grade in CHC patients, correlated with hepatic injury and can assess DAAs treatment success.

Keywords: HCV; markers of regeneration,necrosis, Sofosbuvir.

\section{Introduction}

$\mathrm{HCV}$ is a major cause of chronic hepatic disease worldwide with a significant variable prevalence, related to the investigated geographic location (Martins et al. 2011).

In chronic $\mathrm{HCV}$ patient, $30 \%$ of cases acquire decompensated liver cirrhosis within 10 years(Thomas and Seeff, 2005). Treatment and eradication of HCV infection with DAAs can improve fibrosis and thus, reduce the diseaserelated morbidity and mortality (Gene et al.,
2012). This was verified by significant regression of the parameters of transient elastography after sustained virologicalresponse (SVR) (Bachofner et al., 2017).

Staging and grading of hepatic fibrosisare fundamentalin $\mathrm{CHC}$ patients not only for monitoring liver illnessadvance, but also for treatment indication (Castera, 2011).Liver biopsy was the gold standard for assessing liver fibrosis grade. However; owing to its invasiveness, specialists and patients favor non-invasive tests for fibrosis staging as Fibroscan as it is a safe, rapid, but it is

Copyright: () Alnoby et al. (2021) Immediate open access to its content on the principle that making research freely available to the public supports a greater global exchange of knowledge. Users have the right to Read, download, copy, distribute, print or share link to the full texts under 
expensive and may be not always available (El-Hariri et al., 2017).

Many non-invasive tests based on measurement of direct or indirect serum markers have been developed. Almost all of them have the ability to identify significant fibrosis and cirrhosis in particular (Saad, 2014).Serum liver cell necrosis markersinclude theincreasesin transaminases as aspartate aminotransferase/alanine aminotransferase (ALT/AST), total bilirubin, lactate dehydrogenase, $Y$-glutamyl transferase (GGT) activity, bile acids, alkaline phosphatase, and liver regeneration markers include RBP4, gamma-glutamyl transferase, AFP, and des-gamma-carboxyprothrombin(Kevin et al., 1999).

Fibrosis calculated scores and indices are simple, inexpensive, noninvasive tests used in defining the degree of fibrosis, they include FIB4, APRI, CDC, GUCI and LOK Index (Bonacini et al., 1998; Islam et al., 2005; Lok et al., 2005; Wai et al., 2003; ValletPichard et al., 2007).

\section{Materialsand Method \\ Study design: A prospective, case-control, hospital based study carried out on HCV patients attending; Gastroenterology and Hepatology and Infectious Diseases Department out-patient clinic in Qena university hospital from Jan. 2018 to Dec. 2018.}

Sample size:50 HCV patients and 20 healthy age and sex-matched subjects as controls.

\section{Target population}

Inclusion criteria:treatment naïve patients, age older than 18 years with HCV RNA positivity. Exclusion criteria:

Gestation, acutehepatitis, or other forms of hepatic disorders such ashepatitis-B and HIV infection, Bilharziasis, auto-immunehepatitis, DM, drugs addiction or alcohol abuse, hepatocellular carcinoma, evidence of liver cell failure, cardiac cirrhosis or 1ry biliary cirrhosis or hereditary hepatic disorders.
The study protocol was approved by the local institutional ethics committee and all patients gave their written consent prior to the study.

\section{Methodology}

All of the patients and controls were subjected to $10 \mathrm{ml}$ fasting venous blood sampling,obtained under aseptic conditions into 3 tubes; tube for complete blood count, tube for prothrombin time, and plain tubes for liver functions and ELISA assays.Serumwere gained by centrifugation of the clotted blood at $3000 \mathrm{x} \mathrm{g}$ for $10 \mathrm{~min}$ at room temp, and allocated and kept at $-80^{\circ} \mathrm{C}$ till the time of RBP4 assay.

Full blood count: using cell dyne-1800 (Abbott Diagnostics - SantaClaraCalifornia-USA).

Liver function assessment:ALT (0-41 $\mathrm{U} / \mathrm{L})$, and AST, (0-38 U/L), alkaline phosphatase (ALP), lactate dehydrogenase (LDH)], $\gamma$-glutamyl transferase, albumin, total bilirubin, glucose $(70-100 \mathrm{mg} / \mathrm{dl})$, urea $(10-50 \mathrm{mg} / \mathrm{dl})$, and creatinine $(0.50-1.2$ $\mathrm{mg} / \mathrm{dl}$ ), using Cobas c311 (Roche Diagnostics, Mannheim-Germany). Prothrombin time (PT) using STA Compact Max® Coagulation System (Stago-USA). Hepatitis serumtesting for hepatitis B ( $\mathrm{sAg}$ ) and $\mathrm{HCV}$ antibody detected by an enzyme immunoassay (EIA) Cobas e411 (Roche Diagnostics, Mannheim- Germany).

Alphafetoprotein (AFP): assayedbased on the manufacturer's directives, by an automated immunoassay-Tosoh Bioscience(ST.AIA-PACK ${ }^{\mathrm{TM}}$ AFP Cat. No; 0025252) (Tosoh Corporation Minato-kuTokyo- Japan). The adult reference range $(<$ $7.0 \mathrm{ng} / \mathrm{ml}$ ) and the coefficient of variation was $\leq 5 \%$ across the linear range (1-1210 $\mathrm{ng} / \mathrm{ml}$ ) of measurement and the minimal measurable concentration (MDC) was 1.0 $\mathrm{ng} / \mathrm{ml}$. The standard level was $(0-10 \mathrm{ng} / \mathrm{ml})$.

\section{Serum retinol-binding protein 4} (RBP4):assayed according to the manufacturer's guidelinesusing ELISA Kit Cat. No.E0929h-EIAab ${ }^{\circledR}$ Science Co. Ltd. Wuhan-China. The MDC $0.0510 \mathrm{ng} / \mathrm{ml}$ and the finding range was $0.156-10.0 \mathrm{ng} / \mathrm{ml}$. 
Liver fibrosisextent:by Transient Elastography (TE) Fibroscan (Echosens, FS502 touch device, Paris, France).normal values $(2.5$ to $7.0 \mathrm{kPa})$. subjectsdivided into subgroups accoedingto the optimal cutoff value of liver fibrosis ranges 2.0 to $7.0 \mathrm{kPa}=$ F0-F1 (no/mildhepatic scarring), 8.0 to $9.0 \mathrm{kPa}=\mathrm{F} 2$ (Moderate hepatic scarring), 9.0 to $14.0 \mathrm{kPa}=\mathrm{F} 3$ (Severe hepatic scarring), $14 \mathrm{kPa}$ or higher $=\mathrm{F} 4$ (advanced hepatic scarring or cirrhosis). The calculated computed scores were made according to published formulas APRI (Wai et al., 2003), Cirrhosis discriminate score (CDS) (Bonacini et al., 1998), FIB-4(ValletPichard et al., 2007), GUCI (Islam et al., 2005), and Lok index (Lok et al., 2005).

Statistical analyses:All analyses were performed with the IBM SPSS 20.0 software. The data were tested for normality using the Anderson Darling test and for homogeneity variances prior to further statistical analysis. Categorical variables were described by number and percent $(\mathrm{N}, \%)$, where continuous variables described by mean and standard deviation (Mean, SD). Chi-square used for comparison between categorical variables, whereas independent samples $\mathrm{t}$ test and paired $\mathrm{t}$ test used to compare between continuous variables. Receiver operating characteristic (ROC) curves were constructed to determine the optimal cutoff values for each bio-marker. The accuracy and the diagnostic performance of noninvasive fibrosis tests were compared with the area under the receiver operating curve (AUROC) and their corresponding 95\% confidence intervals (CI) and the sensitivity, specificity, positive predictive value (PPV), negative predictive value (NPV), were calculated. The diagnostic accuracy and AUROC were calculated based on TE
Fibroscan as a reference. All calculation is two-tailed, $\mathrm{p}<0.05$ was considered statistically significant. Pearson correlation was used to detect the association between variables.

\section{Results}

Demographic data: 50 chronic $\mathrm{HCV}$ patients (28 males and 22 females) with a mean age $(55.66 \pm 10.49$ years $)$, BMI (25.27 \pm 2.1$)$. All patients were Child-Pugh score A Mean \pm SD (5.12 \pm 0.33$)$, where 45 patients received (SOF/DAC) therapy and 5 patients received (SOF/DAC/RBV) therapy. The mean PCR HCV viral load was (1449308.18 \pm 1114712.26 ). All treated patientsachievedSVR. The control group hada mean age of 54.60 \pm 3.56 years (11 were males and 9 females) and their BMI was $25.74 \pm 3.45 \mathrm{~kg} / \mathrm{m}^{2}$.

Laboratory data: compared to the control group,patients had higherliver enzymes, INR, AFP, and fibrosis scores (CDS, Lok index, Fib-4 score, GUCI and APRI), and lower albumin, total protein, RBP4, hemoglobin and platelet count. All parameters showedenhancement after therapy (improvement of liver function and reduction of liver enzymesand fibrosis by Fibroscan), Table1.

Correlations with fibroscan result in CHC patients: TE fibroscan results are positively correlated with fibrosis scores and indices (CDS, Lok index, Fib-4 score, GUCI and APRI), INR, MPV, AST and total bilirubin. And negatively correlated with RBP4, Hb, RBCs count, platelet count, albumin, total protein, Eosinophiles percentage and absolute count, and lymphocyte absolute count, Table 2. 
Table 1: laboratory findings in patients and controls:

\begin{tabular}{|l|l|l|l|l|l|l|}
\hline \multirow{2}{*}{ Parameter } & \multicolumn{2}{|c|}{ Cases $(\mathrm{n}=50)$} & \multirow{2}{*}{ ontrol $(\mathrm{n}=20)$} & P1 & P2 & P3 \\
\cline { 2 - 7 } & \multicolumn{1}{|c|}{ Pre } & Post & & & & \\
\hline Fibroscan result kPa & $9.15 \pm 2.65$ & $7.96 \pm 2.31$ & - & 001 & - & - \\
\hline CDS & $5.1 \pm 1.78$ & $4.54 \pm 1.39$ & $2.65 \pm 0.88$ & 065 & 001 & 001 \\
\hline LOK INDEX & $0.44 \pm 0.22$ & $0.33 \pm 0.18$ & $0.15 \pm 0.07$ & 003 & 001 & 001 \\
\hline GUCI & $1.03 \pm 1.1$ & $0.43 \pm 0.28$ & $0.12 \pm 0.05$ & 001 & 001 & 118 \\
\hline APRI & $0.93 \pm 0.97$ & $0.4 \pm 0.24$ & $0.12 \pm 0.05$ & 001 & 001 & 101 \\
\hline FIB-4 & $2.64 \pm 1.84$ & $1.58 \pm 0.84$ & $0.68 \pm 0.18$ & 001 & 001 & 011 \\
\hline Albumin $(\mathrm{g} / \mathrm{dl})$ & $3.85 \pm 0.48$ & $3.95 \pm 0.42$ & $4.13 \pm 0.16$ & 240 & 015 & 120 \\
\hline Total Protein $(\mathrm{g} / \mathrm{dl})$ & $7.27 \pm 0.59$ & $7.47 \pm 0.5$ & $8.06 \pm 0.34$ & 052 & 001 & 001 \\
\hline ALT $(\mathrm{U} / \mathrm{L})$ & $9.48 \pm 48.28$ & $3.1 \pm 15.44$ & $15.15 \pm 6.52$ & 001 & 001 & 041 \\
\hline AST $(\mathrm{U} / \mathrm{L})$ & $1.78 \pm 56.46$ & $4.92 \pm 17.3$ & $15.4 \pm 5.58$ & 001 & 001 & 056 \\
\hline T. BIL $(\mathrm{mg} / \mathrm{dl})$ & $0.95 \pm 0.31$ & $0.73 \pm 0.27$ & $0.57 \pm 0.13$ & 001 & 001 & 022 \\
\hline ALP $(\mathrm{U} / \mathrm{L})$ & $10.3 \pm 42.93$ & $96 \pm 33.49$ & $48.45 \pm 8.54$ & 046 & 001 & 001 \\
\hline GGT $(\mathrm{U} / \mathrm{L})$ & $2.28 \pm 12.18$ & $25.3 \pm 10.7$ & $15.35 \pm 5.84$ & 002 & 001 & 001 \\
\hline LDH $(\mathrm{U} / \mathrm{L})$ & $7.96 \pm 33.89$ & $8.02 \pm 28.33$ & $16.45 \pm 13.54$ & 001 & 001 & 001 \\
\hline AFP $(\mathrm{ng} / \mathrm{mL})$ & $8.52 \pm 2.87$ & $4.89 \pm 2.2$ & $2.05 \pm 0.62$ & 001 & 001 & 001 \\
\hline RBP4 $(\mathrm{mg} / \mathrm{l})$ & $38.34 \pm 2.71$ & $5.83 \pm 2.12$ & $55.17 \pm 4.54$ & 001 & 001 & 001 \\
\hline INR & $1.08 \pm 0.14$ & $1.04 \pm 0.08$ & $1.01 \pm 0.01$ & 036 & 008 & 273 \\
\hline PLT $\left(10^{3} / \mathrm{mm}^{3}\right)$ & $7.24 \pm 80.19$ & $35.4 \pm 73.84$ & $28.65 \pm 59.24$ & 012 & 001 & 001 \\
\hline WBC $\left(10^{3} / \mathrm{mm}^{3}\right)$ & $5.88 \pm 1.85$ & $7.15 \pm 1.98$ & $6.52 \pm 2.38$ & 002 & 231 & 230 \\
\hline
\end{tabular}

P1: Pre VS Post; P2: Pre VS Control; P3: Post VS Control

To differentiate mild fibrosis (F0-1) from significant fibrosis (F2-F3-F4):ROC curve showed that Fib-4 score is more superior subsequently APRI and GUCI. As the accuracy of Fib-4 produced (AUROC 0.985, 95\% CI 83.8-99.4). And the accuracy of APRI and GUCI both produced (AUROC of $0.948,95 \%$ CI 77.4 - 97.3). The accuracy of CDC produced (AUROC 0.893, 95\% CI 87.4-99.9). The accuracy of LOK index produced (AUROC $0.921,95 \%$ CI $91.6-100)$. The accuracy of RBP4 produced (AUROC $0.811,95 \%$ CI 77.4 97.3). For definitive diagnosis of significant fibrosis (i.e. $100 \%$ specificity and $100 \%$ PPV), using APRI, Fib-4 and GUCI at the cutoff value of $0.35,1.17$, and 0.37 respectively, Figure 1.

To differentiate (F0-1-2) versus severe fibrosis (F3-4): the diagnostic performance of FIB4 is the most superior (AUROC 0.940, 95\% CI 69.8 - 99.8). Followed by RBP4 (AUROC
0.93, 93.75\% CI 69.8-99.8), followed by GUCI and LOK index both produced (AUROC 0.935 and 0.882 respectively, 95\% CI $47.6-92.7$ ), Figure 2.

Table 2: Correlation with fibroscan result:

\begin{tabular}{|l|c|c|}
\hline \multirow{2}{*}{} & \multicolumn{2}{|c|}{ Fibroscan result kPa } \\
\cline { 2 - 3 } & $\mathbf{R}$ & $\mathbf{P}$ \\
\hline Age & 0.172 & 0.232 \\
\hline BMI & 0.038 & 0.793 \\
\hline CDS & $0.850^{* *}$ & 0.001 \\
\hline LOK INDEX & $0.837^{* * *}$ & 0.001 \\
\hline GUCI & $0.595^{* * *}$ & 0.001 \\
\hline APRI & $0.560^{* * *}$ & 0.001 \\
\hline FIB-4 & $0.765^{* *}$ & 0.001 \\
\hline Albumin (g/dl) & $-0.336^{* *}$ & 0.017 \\
\hline Total Protein (g/dl) & $-0.457^{* *}$ & 0.001 \\
\hline ALT (U/L) & 0.245 & 0.086 \\
\hline AST (U/L) & $0.388^{* *}$ & 0.005 \\
\hline T. BILT (mg/dl) & $0.323^{*}$ & 0.022 \\
\hline ALP (U/L) & 0.177 & 0.218 \\
\hline GGT (U/L) & 0.218 & 0.129 \\
\hline
\end{tabular}




\begin{tabular}{|l|c|c|}
\hline \multirow{2}{*}{} & \multicolumn{2}{|c|}{ Fibroscan result kPa } \\
\cline { 2 - 3 } & \multicolumn{1}{|c|}{$\mathbf{R}$} & $\mathbf{P}$ \\
\hline LDH $(\mathrm{U} / \mathrm{L})$ & -0.009 & 0.948 \\
\hline AFP $(\mathrm{ng} / \mathrm{mL})$ & 0.187 & 0.192 \\
\hline RBP4 $(\mathrm{mg} / \mathrm{l})$ & $-0.777^{* *}$ & 0.001 \\
\hline PCR $(\mathrm{IU} / \mathrm{ml})$ & 0.053 & 0.715 \\
\hline INR & $0.416^{* *}$ & 0.003 \\
\hline PLT $\left(10^{3} / \mathrm{mm}^{3}\right)$ & $-0.74-^{* *}$ & 0.001 \\
\hline MPV & $0.353^{*}$ & 0.012 \\
\hline WBC $\left(10^{3} / \mathrm{mm}^{3}\right)$ & -0.138 & 0.338 \\
\hline
\end{tabular}

*Statistically significant correlation $<0.05$

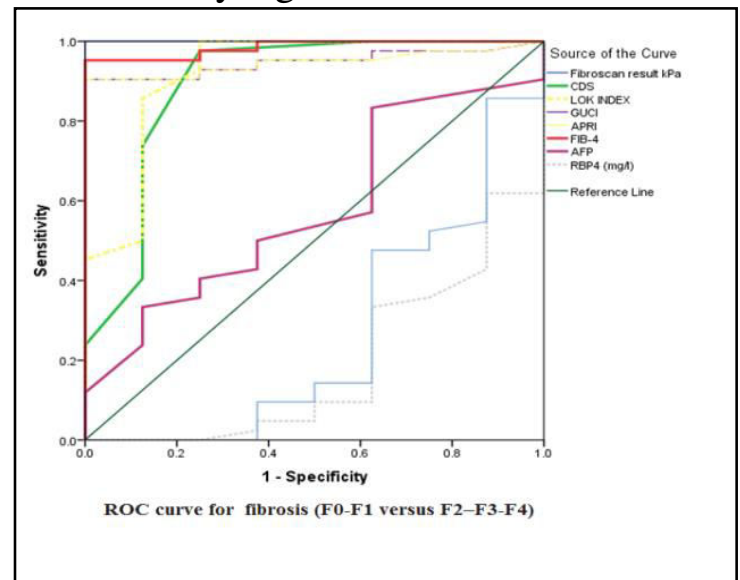

Figure 1: ROC curve for significant fibrosis(F0- F1 versus F2-F3-F4)

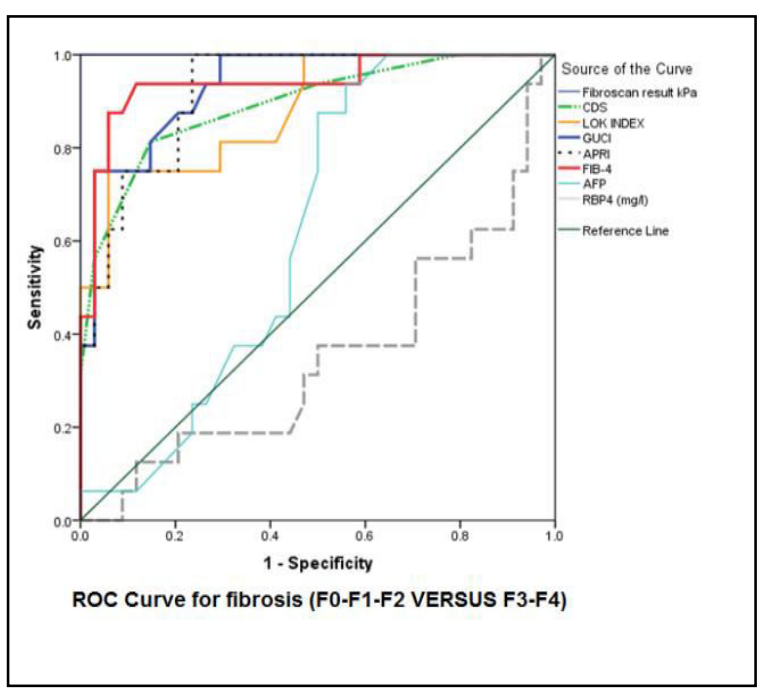

Figure 2:ROC curve for significant fibrosis (F0-F1-F2 versus F3-F4)

\section{Discussion}

Eradication of HCV infection is avital medical seek in Egypt so as to avoid progression of liver fibrosis and later cirrhosis and its complications, including liver cell failure and portal hypertension (Suo and Zhao, 2013).
Over the past several years, all oral DAAs agents have replaced IFN for the treatment of HCV.The advantages of DAAs improve tolerability, safety and utility and patients with advanced cirrhosis who were not candidates for antiviral therapy before could now be treated by DAAs (Elkassas et al., 2017).

In this study; wenoted a significantincrease in liver enzyme levels and fall in the hepatic synthetic function (decreased serum albumin, RBP4, and platelet count and increased INR). This was in line with (Allam et al., 2015).

The patients had an elevated AFP compared to controls, with a noteworthydecline after treatment and SVR achievement, reflecting regression in disease activity. This was in line with (Allam et al., 2015 and Deterding et al., 2015).

Also in this study, we found a significant recession in fibroscan value in all patients both at the end of treatment and 6 months post-treatment from $9.15 \pm 2.65 \mathrm{kPa}$ to $7.96 \pm 2.31 \mathrm{kPa}$. This finding goes in line with (Bachofner et al., 2017)whofound that patients with SVR showed marked improvement in the median transient elastography (TE) values from $12.65 \mathrm{kPa}$ to $8.55 \mathrm{kPa}$ after DAA therapy.

The prognosis and management of chronic liver diseases largely depends on the extent and the progress of liver fibrosis. Histopathological check of a liver specimen obtained by percutaneous biopsy has traditionally been considered as the gold standard for evaluating hepatic fibrosis (Bravo et al., 2001). However, liver biopsy is an invasive procedure, had poor patient acceptance and also carries a significant, although small risk of lifethreatening complications(Cadranel et al., 2000). So to overcome liver biopsy limits, several non-invasive blood tests developed to assess hepatic fibrosis. In this study, we investigated five non-invasive indices that can reliably predict the histological stage of liver fibrosis/cirrhosis (APRI, CDS, Fib-4, GUCI, Lok index) (Hsieh et al., 2012) 
The comparison of the five noninvasive fibrosis indices and scores showed that, with the rising severity of liver fibrosis (evaluated by TE Fibroscan); their values increased significantly together with the increase of serum AFP and the decrease of serum albumin, RBP4, INR and platelet count. This suggests that they are a good estimator of hepatic fibrosis and can all effectively evaluate accurately different stages of liver fibrosis in $\mathrm{CHC}$ patients and could be used to decrease the number of liver biopsies. This was in line with (Amorim et al., 2012; Baranova et al., 2011;Derbala et al., 2015; Khan et al., 2008; Li et al., 2014; Snyder et al., 2006).

In this study, the viral load correlated positively with TE Fibroscan and AFP and correlated negatively with RBP4, while RBP4 showed a positive correlation with platelet count, and a negative correlation with fibrosis stage, viral load, AFP, and the five non-invasive fibrosis indices (APRI, CDS, Fib-4, GUCI, Lok index). This was in agreement with the findings of (Derbala et al., 2015; Li et al., 2014).

In this study, in CHC, the Fib-4 score (as estimated by ROC curve) gave the greatest performance and investigative accuracy inthe prediction of significant fibrosis, then (APRI and GUCI) followed by the CDC and LOK index; and lastly RBP4 as they were efficient to differentiate significant fibrosis (moderate and severe) liver scarring (F2-F3) from mild fibrosis (F0-F1). This was in line with (Bonacini et al., 1998; Islam et al., 2005; Lok et al., 2005; Wai et al., 2003; Vallet-Pichard et al., 2007). However; the accuracy of RBP4 was improved with a higher grade of fibrosis and thus can differentiate the presence or absence of severe liver scarring (F3-F4) from mild \& moderate (F1-F2) liver scarring with $93.75 \%$ for PPV and NPV but with a poor prediction of the absence of significant fibrosis (F1 versus F2-F3). Furthermore, LOK score was excellent in the prediction of the absence of significant fibrosis (F1 versus F2-F3). AFP showed a positive correlation with fibrosis stage, viral load, four non-invasive fibrosis indices (APRI, Fib-4, GUCI and Lok index), and had a negative correlation with RBP4. In contrast, Tai et al.
(2009) found that AFP levels were positively correlated with the age, ALT, fibrosis grade, and were negatively correlated with platelet count. However, in our study, it has been found to have poor judgment power to differentiate the different stages of liver fibrosis.

In this study, CHC patient had a significant decline in the mean RBP4 after treatment. This was in line with (Kataria et al., 2016). RBP4 correlatedpositively with platelet count and inversely correlated with the disease severity and fibrosis score, viral load, AFP, the five noninvasive fibrosis indices (APRI, CDS, Fib-4, GUCI, and Lok index), asthe serum level of retinol depends on the hepatic synthesis and excretion of RBP4 that, in turn, decreases with increased liver damage (Peres et al., 2011).

For the diagnosis of significant fibrosis, TE and serum biomarkers from routine blood tests were equivalent. So to raise the diagnostic accuracy, a mixture of TE and serological markers of the non-invasive fibrosis indices was recommended by using an algorithm starting with a screening test using noninvasive fibrosis indices and restricting $\mathrm{TE}$ or liver biopsy in patients classified as $\mathrm{F} 0-\mathrm{F} 1$ by noninvasive serological tests, (Boursier et al., 2009; Castera et al. 2005; Poynard et al., 2008; Sebastiani et al., 2006).

The limitations of this study included the small number of patients and the exclusion of patients with concomitant liver disease, thus potentially limiting the generality of our results to other populations.

\section{Conclusion}

In CHC patients, serological tests for fibrosis indices have a good accurate diagnostic value that can predict the occurrence or absence of significant fibrosis and cirrhosis. They are cost-free,available, easy to calculate and reproducible indices. The use of the combination of these tests may avoid the need for invasive or expensive tests as liver biopsy and fibroscan. Serum RBP4 is a good marker for the prediction of definite and severe fibrosis and its rise after successful 
$\mathrm{HCV}$ eradication is considered a marker of stoppage and reduction of inflammation.

\section{References}

Allam A, Gabr S, Ajarem J, AbdelMaksoud M.(2015).Bcl-2 and p53 expression in hepatic tissues of Egyptian patients with Chronic Hepatitis C. J Pak Med Assoc, 65(11):1186-92.

Amorim TG, Staub GJ, Lazzarotto C, Silva AP, Manes J, FerronatoMda G, Shiozawa MB, Narciso-Schiavon JL, Dantas-Correa EB. (2012). Schiavon Lde L. Validation and comparison of simple noninvasive models for the prediction of liver fibrosis in chronic hepatitis C. Ann Hepatol, 11: 855-861.

Azzazi MO, Mohamed MA, Mousa MM, Mohammed RM, Eldin Youssef SS.(2017)Multicentre study of hepatitis C virus status in Egyptian patients with B-cell non-Hodgkin's lymphoma with assessment of patients' immunological state. Egypt J Haematol, 42:19-30.

Bachofner JA, Valli PV, Kröger A, Bergamin I, Künzler P, Baserga A, Braun D, Seifert B, Moncsek A, Fehr J, Semela D, Magenta L, Müllhaupt B, Terziroli BerettaPiccoli B, Mertens JC.(2017).Direct antiviral agent treatment of chronic hepatitis $\mathrm{C}$ results in rapid regression of transient elastography and fibrosis markers fibrosis- 4 score and aspartate aminotransferase- platelet ratio index. Liver Int, 37(3):369-376.

Baranova A, Lal P, Birerdinc A, Younossi ZM.(2011). Non-invasive markers for hepatic fibrosis. BMC Gastroenterol, 11: 91.

Bonacini M, Hadi G, Govindarajan S, Lindsay K.(1997). Utility of a discriminant score for diagnosing advanced fibrosis or cirrhosis in patients with chronic hepatitis $\mathrm{C}$ virus infection. Am J Gastroenterol., 92(8):1302-4.
Boursier J, Vergniol J, Sawadogo A, Dakka T, Michalak S, Gallois Y, et al. (2009). The combination of a blood test and Fibroscanimproves the non-invasive diagnosis of liver fibrosis. Liver Int., 29:1507-1515.

Bravo AA, Sheth SG, Chopra S.(2001).Liver Biopsy. N Engl J Med, 344: 495-500.

Cadranel JF, Rufat P, Degos F.(2000). Practices of Liver Biopsy in France: Results of a Prospective Nationwide Survey. Hepatology, 32(3):477-81.

Castera L, Vergniol J, Foucher J, Le Bail B, Chanteloup E, Haaser M, et al.(2005). Prospective comparison of transient elastography, Fibrotest, APRI, and liver biopsy for the assessment of fibrosis in chronic hepatitis C. Gastroenterology, 128:343-350.

Castera L. (2011). Noninvasive assessment of liver fibrosis in chronic hepatitis $C$. HepatolInt, 5:625-634.

Derbala M, Elbadri ME, Amer AM, AlKaabi S, Sultan KH, Kamel YM, Elsayed EH, Avades TY, Chandra P, Shebl FM.(2015). Aspartate transaminase to platelet ratio index in hepatitis $\mathrm{C}$ virus and Schistosomiasis coinfection.World J.Gastroenterol, 21(46):13132-9.

Deterding K, HönerZuSiederdissen C, Port K, Solbach P, Sollik L, Kirschner J, Mix C, Cornberg J, Worzala D, Mix H, et al.(2015). Improvement of liver function parameters in advanced $\mathrm{HCV}$-associated liver cirrhosis by IFN-free antiviral therapies. Aliment PharmacolTher., 42:889-901.

El Kassas M, El-Akel W, El-Sayed MH, El-Serafy M, Khairy M, Elsaeed K, Kabil K, Hassany M, Shawky A, Yosry A, Shaker MK, EIShazly Y, Waked I, Esmat G, Doss W.(2017).National treatment programme of hepatitis $\mathrm{C}$ in Egypt: Hepatitis $\mathrm{C}$ virus model of care. Journal of Viral Hepatitis, 24(4):262267. 
El-Hariri M, Abd El Megid AG, Hassany M.(2017). Diagnostic value of Transient Elastography (Fibroscan) in the evaluation of liver fibrosis in chronic viral hepatitis $C$ : Comparison to liver biopsy. The Egyptian Journal of Radiology and Nuclear Medicine, 48(2):329-337.

Gene Y, Douglas T.(2012).Dieterich. DirectActing Antiviral Agents in Patients with Hepatitis C Cirrhosis.GastroenterolHepatol, (N Y). 8(11): 727-765.

Hsieh YY, Tung SY, Lee K, Wu CS, Wei KL, Shen CH, Chang TS, Lin YH.(2012). Routine blood tests to predict liver fibrosis in chronic hepatitis C. World J Gastroenterol, 18:746-53.

Islam S, Antonsson L, Westin J, Lagging M.(2005). Cirrhosis in hepatitis $C$ virusinfected patients can be excluded using an index of standard biochemical serum markers. Scand J Gastroenterol, 40:867-872.

Kataria Y, Deaton RJ, Enk E, Jin M, Petrauskaite M, Dong L, Goldenberg JR, Cotler SJ, Jensen DM, van Breemen RB, Gann PH.(2016). Retinoid and carotenoid status in serum and liver among patients at high-risk for liver cancer. BMC Gastroenterology, 16:30.

Kevin D Horn, Paul Wax, Sandra M Schneider, Thomas G Martin, Jeffrey S Nine, Michael A Moraca, Mohamed A Virji, Patricia A Aronica, Kalipatnapu N Rao.(1999).Biomarkers of liver regeneration allow early prediction of hepatic recovery after acute necrosis. AJCP , 112(3): 351 357.

Khan DA, Fatima-Tuz-Zuhra FA, Mubarak A.(2008). Evaluation of diagnostic accuracy of APRI for prediction of fibrosis in hepatitis C patients. J Ayub Med Coll Abbottabad, 20: 122-126.
Li SM, Li GX, Fu DM, Wang Y, Dang LQ.(2014).Liver fibrosis evaluation by ARFI and APRI in chronic hepatitis C. World J Gastroenterol, 20: 9528-9533.

Lok AS, Ghany MG, Goodman ZD, Wright EC, Everson GT, Sterling RK, et al.(2005).Predicting cirrhosis in patients with hepatitis $\mathrm{C}$ based on standard laboratory tests: results of the HALT-C cohort. Hepatology, 42:282-292.

Martins T, Narciso-Schiavon JL, Schiavon LL.(2011).Epidemiology of hepatitis $C$ virus infection. Rev Assoc Med Bras, 57(1): 10510.

Peres WA, Chaves GV, Gonc alves JCS, Ramalho A, Coelho HS.(2011). Vitamin A deficiency in patients with hepatitis $C$ virusrelated chronic liver disease. $\mathrm{Br} \mathrm{J}$ Nutr., 106(11):1724-1731.

Poynard T, Ingiliz P, Elkrief L, Munteanu M, Lebray P, Morra R, et al.(2008).Concordance in a world without a gold standard: a new non-invasive methodology for improving accuracy of fibrosis markers. PLoS ONE, 3:e3857.

Saad EA.(2014).Noninvasive Assessment of Liver Fibrosis Using Serum Markers, J. Pharm ChemBiolSci, 2(2):59-76.

Sebastiani G, Vario A, Guido M, Noventa F, Plebani M, Pistis R, Ferrari A, Alberti A.(2006). Stepwise combination algorithms of non-invasive markers to diagnose fibrosis in chronic hepatitis C. J.Hepatol, 44(4): 68669.

Snyder N, Gajula L, Xiao SY, Grady J, Luxon B, Lau DT, Soloway R. (2006).Petersen J. APRI: an easy and validated predictor of hepatic fibrosis in chronic hepatitis C. J ClinGastroenterol, 40: 535-542.

Suo GJ, Zhao ZX.(2013). Association of the interleukin-28B gene polymorphism with 
development of hepatitis virus-related hepatocellular carcinoma and liver cirrhosis: a meta-analysis. Genet. Mol. Res. 12(3): 3708-3717 .

Tai WC, Hu TH, Wang JH, Hung CH, Lu SN, Changchien CS, Lee CM.(2009). Clinical Implications of Alpha-fetoprotein in Chronic Hepatitis C. J Formos Med Assoc 108(3):210-218.

Thomas DL, Seeff LB.(2005).Natural history of hepatitis C. Clin Liver Dis, 9:383398.
Vallet-Pichard A, Mallet V, Nalpas B, Verkarre V, Nalpas A, Dhalluin-Venier V, Fontaine H, Pol S.(2007). FIB-4: an inexpensive and accurate marker of fibrosis in $\mathrm{HCV}$ infection. Comparison with liver biopsy and fibro test. Hepatology, 46:32-36.

Wai CT, Greenson JK, Fontana RJ, Kalbfleisch JD, Marrero JA.(2003).Conjeevaram HS, Lok AS. A simple noninvasive index can predict both significant fibrosis and cirrhosis in patients with chronic hepatitis C. Hepatology,38(2):518-526. 